\title{
Preconception immunization can modulate intracellular Th2 cytokine profile in offspring: in vivo influence of interleukin 10 and $B / T$ cell collaboration
}

\author{
MARÍLIA GARCIA DE OLIVEIRA', ALINE APARECIDA DE LIMA LIRA ${ }^{l}$, \\ FÁBIO DA RESSUREIÇĂO SGNOTTO ${ }^{l}$, AMANDA HARUMI SABÔ INOUE', \\ ALBERTO JOSÉ DA SILVA DUARTE ${ }^{1,2}$, JEFFERSON RUSSO VICTOR ${ }^{1,2,3}$ \\ ${ }^{1}$ Laboratory of Medical Investigation LIM 56, Division of Clinical Dermatology, Medical School, University of Sao Paulo, Sao Paulo, Brazil \\ ${ }^{2}$ Division of Pathology, Medical School, University of Sao Paulo, Sao Paulo, Brazil \\ ${ }^{3}$ Division of Environmental Health, FMU, Laureate International Universities, Sao Paulo, Brazil
}

\begin{abstract}
Introduction: In the last few years our group has been studying the mechanisms involved in the inhibition of allergy in offspring mediated by preconception maternal immunization, but these mechanisms are not fully understood. Such mechanisms that we have studied aimed at the passive transfer of maternal antibodies and its influence on offspring immune status.

Aim of the study: To evaluate whether maternal immunization could modulate intracellular Th1/ Th2 profiles in offspring.

Material and methods: C57BL/6 female wild type mice (WT), interleukin (IL)-10\% or CD28 $8^{-1}$ mice were immunized or not with ovalbumin (OVA) and were mated with respective lineage males and offspring were evaluated at 3 days old (d.o.), 20 d.o., or 20 d.o. after neonatal immunization.

Results: Preconception OVA immunization induced a marked reduction in IL-4 secretion by TCD4+ cells of WT offspring when compared with offspring from non-immunized mothers. The maternal immunization of $I L-10^{-/}$mice induced an increase in the TCD4+IL-4+ percentage in offspring and a reduction in TCD4+IFN- $\gamma+$ cells. The maternal immunization in CD28- mice induced augment IL-4 intensity in 3 and 20 d.o. offspring TCD4+ cells.

Conclusions: Our results reveal that maternal immunization with OVA can down-regulate the Th2 pattern in offspring and this regulation is dependent on IL-10 and B/T cell collaboration.
\end{abstract}

Key words: allergy, OVA, maternal fetal-interface, IL-10, Th1/Th2 balance, B cells.

(Centr Eur J Immunol 2018; 43 (4): 378-388)

\section{Introduction}

Allergic reactions are characterized by intense Th2 cytokine production profile and exacerbated immunoglobulin $(\mathrm{Ig}) \mathrm{E}$ production. The balance between $\mathrm{Th} 1$ and $\mathrm{Th} 2$ cytokine profiles is regulated mainly by interferon (IFN)- $\gamma$ (Th1-related) and interleukin (IL)-4 (Th2-related) production. The main sources of these cytokines are TCD4 cells but both cytokines can also be produced or regulated by B cells [1].

The prevention strategies to regulate allergy development are classified into three levels, with the primary strategy aiming to prevent allergen sensitization, the secondary aiming at the prevention of allergy development in already sensitized individuals, and tertiary aiming at the treatment of the symptoms [2]. Primary strategies have been studied by our group in type I hypersensitivity murine models over the last 15 years [3-12]. These models consider three important factors: the maternal immunity during pregnancy, neonatal immunity, and the interaction between the two. The maternal and fetal immune system interaction occurs either through the placenta or through breast-feeding. Previously, we reported that maternal immunization with the dust mite Dermatophagoides pteronyssinus (Dp) can inhibit offspring Th2 exacerbation [13] characteristics that was also observed after maternal immunization with OVA [14]. In the same work, we also reported that the passive transfer of maternal IgG can modulate offspring IL-10 pro-

Correspondence: Jefferson Russo Victor, PhD, Laboratory of Medical Investigation LIM-56, Division of Clinical Dermatology,

Medical School, University of Sao Paulo, Sao Paulo, Brazil, Av. Dr. Enéas de Carvalho Aguiar, 500, $3^{\text {rd }}$ floor, 05403-000 Săo Paulo, Brazil, phone: +55 11 30617499, fax: +55 1130817190 , e-mail: victorjr@usp.br Submitted: 19.05.2017; Accepted: 16.10.2017 
duction, but at the time we did not evaluate the impact of IL-10 upon this mechanism using IL-10 ${ }^{-/}$mice.

The B cells that produce IL-10 (B10) have their regulatory function dependent on IL-4 production [15], an important Th2 pattern cytokine. Amu and co-workers noted that B10 cells could prevent and reverse allergic airway inflammation in a similar murine model of immunization with OVA in BALB/c mice [16]. Furthermore, B10 cells can participate in the regulation of $\mathrm{IgE}$ production, as shown in an atopic dermatitis-like mouse model [17] and induce regulatory cells, such as $\operatorname{Tr} 1$ and nTreg [18].

Recently our group has demonstrated, in the same experimental model used in the present work, that maternal preconception immunization with OVA can induce B10 cells in offspring [19], but the effect of maternal immunization upon $\mathrm{B}$ and $\mathrm{T}$ cells cytokine secretion pattern was not evaluated at the time.

Since our experimental protocol immunizes mothers with allergens, another question that emerges from our results is whether the induction of $\operatorname{IgE}$ and $\mathrm{Th} 2$ profile needs to be induced in mothers to protect offspring from allergy development. To elucidate this question the use of CD28mice can be very useful. These animals are unable to produce $\operatorname{IgE}$ in response to allergen sensitization, as shown by the Th1-driven immunity with the predominance of IFN- $\gamma$ production, and because its $\mathrm{B} / \mathrm{T}$ cell cooperation is compromised [20]. Based on this, the present work aims to evaluate, in a complementary way to the data already published by our group, the impact of maternal OVA immunization upon offspring IFN- $\gamma$ (Th1-related) and IL-4 (Th2-related) intracellular production on two main resources of cytokines, TCD4 and B cells. Together with previous results, the understanding of these mechanisms may contribute to the development of therapeutic strategies for allergy inhibition in offspring through maternal immunization.

\section{Material and methods}

\section{Mice}

C57BL/6 inbred wild type mice (WT) or IL-10 and CD28 genetically deficient (IL-10 ${ }^{-/}$and CD28 $8^{-/}$) males and females were used at 8-10 weeks old. The animals were purchased through the Central Animal Facility of the School of Medicine and Institute of Biomedical Sciences - USP. The offspring (F1) of both sexes were used. All experiments described in this manuscript were approved by the University of Sao Paulo School of Medicine Animal Ethics Committee (CEP-FMUSP: 122/14 - Sao Paulo, SP, Brazil).

\section{Murine immunization}

Female WT mice were immunized subcutaneously with $1500 \mu \mathrm{g}$ of OVA (Sigma, USA) in $6 \mathrm{mg}$ of aluminum hydroxide (FURP, Sao Paulo) and boosted intraperitoneally (ip) after 10 and 20 days with $1000 \mu \mathrm{g}$ of OVA in saline totaling
$200 \mu \mathrm{l}$ per immunization or booster. Females were mated 21 days after immunization. The pups of immunized and non-immune mothers were immunized with the same antigen used for maternal immunization. Three-day-old ( 3 d.o.) offspring were evaluated or treated ip with $100 \mu \mathrm{g}$ of OVA in $0.6 \mathrm{mg}$ of alum (totaling $20 \mu \mathrm{l}$ ), boosted after 10 days with OVA in saline (totaling $200 \mu \mathrm{l}$ ) and evaluated at 20 d.o. [20 (Im) d.o.]. Some groups of animals were not immunized at 3 d.o. and were evaluated at 20 d.o. (20 d.o.). OVA immunization protocols were also performed using $\mathrm{IL}-10^{-/-}$or $\mathrm{CD} 28^{-/-}$mice and are schematically represented in Figure 1.

\section{Spleen cell suspensions}

Spleens were collected, and their cells were isolated for culture or flow cytometry analyses. Single-cell suspensions were prepared with cell strainers (BD Biosciences, MA, USA) and placed in Petri dishes containing RPMI 1640 culture medium (Sigma). The cell suspension was treated with lysis buffer (Biosource - ACK Lysis Buffer, Rockville, MD, USA) for 2 minutes, and the cell suspension was washed twice with RPMI medium. The cells were subsequently resuspended in $1 \mathrm{ml}$ of RPMI medium with $10 \%$ FBS (III HyClone, Logan, USA), and cellular viability was quantified with $0.5 \%$ Trypan blue in a Neubauer chamber.
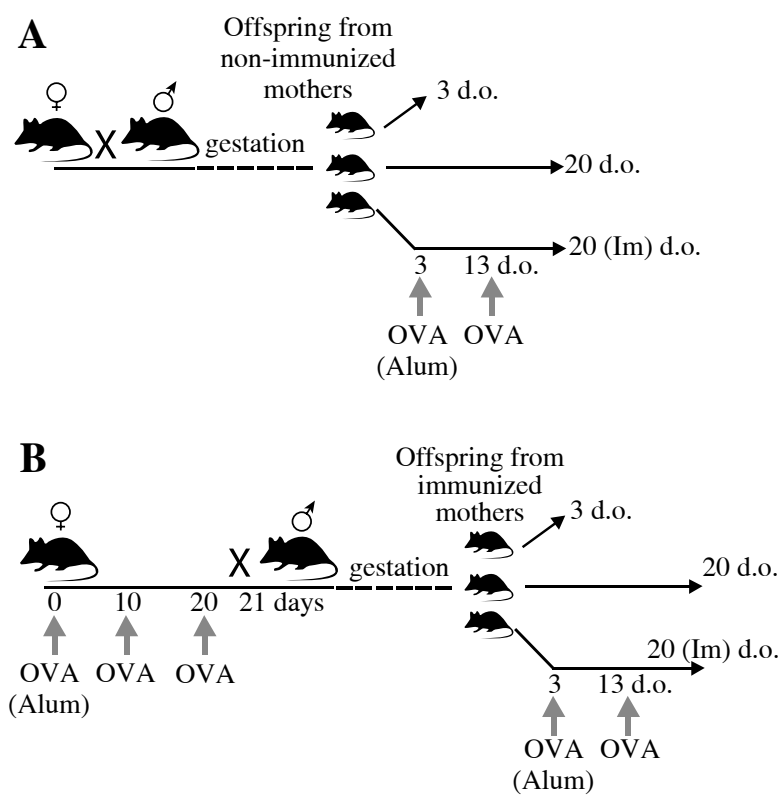

Fig. 1. Schematic representation of experimental protocols used on WT, IL-10 $10^{-/-}$and CD28 $8^{-/-}$mice. WT, IL- $10^{-/-}$or $\mathrm{CD} 28^{-/}$non-immunized females were mated with normal males and offspring were evaluated at 3 d.o., 20 d.o. or immunized with OVA in the neonatal period and evaluated at 20 d.o. (A). OVA immunized WT, IL-10 ${ }^{-/-}$or CD $28^{-/-}$females were mated with normal males and offspring were evaluated at 3 d.o., 20 d.o. or immunized with OVA in the neonatal period and evaluated at 20 d.o. (B) 


\section{Murine flow cytometry}

For surface staining, single-cell suspensions were prepared in flow cytometry buffer (PBS, $1 \%$ BSA). Directly conjugated antibodies with Alexa Fluor 700, PE-Texas Red, FITC, APC or PE (BD Biosciences), anti-CD19 (1D3), anti-B220 (RA3-6B2), and anti-CD4 (RM4-5) were used at optimal concentrations after the titration experiments. Cell gating was based on the specific isotype control values as well as the fluorescence minus one (FMO) to determine intracellular cytokine levels. All analyses were performed using FlowJo software (Tree Star Inc). For intracellular cytokines, cells were cultured for 24 hours at $3 \times 10^{6}$ cells/ml in RPMI 1640 (Gibco) supplemented with $10 \%$ heat-inactivated FCS (HyClone) without stimulus in the presence of $10 \mu \mathrm{g} / \mathrm{ml}$ brefeldin A (Sigma-Aldrich). Cells were first stained for surface markers followed by fixation, permeabilization, and intracellular staining with IFN- $\gamma$ (XMG1.2) or IL-4 (11B11) or matched isotypes using PBS with $0.5 \%$ saponin (Sigma).

Compensation for the instrument was performed using microbeads adsorbed with anti-rat/anti-hamster antibodies (CompBeads - BD Biosciences) and their conjugated antibodies. The acquisition of 200,000 events per sample was performed in the lymphocyte quadrant (as determined by ratio size/granularity) on an LSRFortessa cytometer (BD Biosciences - USA), and analysis was performed using FlowJo software 7.6.5 (Tree Star - USA).

To standardize intracellular cytokine detection, we used as a positive control the stimulus with $10 \mathrm{ng} / \mathrm{ml}$ of phorbol 12-myristate 13-acetate (PMA) and $250 \mathrm{ng} / \mathrm{ml}$ ionomycin for $6 \mathrm{~h}$ [21], and as a negative control we used ex-vivo stained cells. Positive controls induced from 2- to the 5-fold percentage of evaluated cytokines on TCD4 and B cells with a similar mean fluorescence intensity (MFI) levels. In negative controls, the percentage of all evaluated cytokines on TCD4 and B cells was between undetectable and $0.2 \%$; therefore the MFI was not measured.

\section{Statistical analysis and number of samples}

Statistical analysis was performed with GraphPad Prism 5.0 (GraphPad Software Inc., La Jolla, Calif). The data from in vivo and in vitro studies were taken from 3 to 5 separate experiments with 9 to 12 mice per group. Differences were considered significant when $p \leq 0.05$ as assessed by the Mann-Whitney test.

\section{Results}

\section{Maternal immunization regulates offspring Th2 and $B$ cell cytokine production profiles}

The evaluation of the percentage and intensity of cytokine production by offspring TCD4+ cells (Fig. 2) reveals that maternal immunization induced a marked reduction in IL-4 ( $p=0.0337)$ and IFN- $\gamma(p=0.0447)$ intensity at 3 and 20 d.o. offspring from immunized mothers when compared with offspring from non-immunized mothers (Fig. 3B-D). By contrast, the evaluation of the percentage and intensity of cytokine production by offspring B cells (Fig. 4) reveals enhanced IL-4 expression $(p=0.0043)$ as mediated by maternal immunization at 3 d.o. (Fig. 5B), which was maintained until 20 d.o. ( $p=0.0341$, Fig. 5D) when compared with offspring from non-immunized mothers. The percentages of IL-4+ and IFN- $\gamma+$ were not altered in TCD4+ (3 d.o. IL-4, $p=0.1953$ and IFN- $\gamma, p=$ $0.4371 ; 20$ d.o. IL-4, $p=0.4336$ and IFN- $\gamma, p=0.0671$; 20 (Im) d.o. IL-4, $p=0.1255$ and IFN- $\gamma, p=0.9167)$ and B (CD19+; 3 d.o. IL-4, $p=0.2255$ and IFN- $\gamma, p=0.4300$; 20 d.o. IL-4, $p=0.3327$ and IFN- $\gamma, p=0.3391 ; 20$ (Im) d.o. IL-4, $p=0.2752$ and IFN- $\gamma, p=0.3040$ ) cells at any evaluation period (Fig. 3A-C and 5A-C), as well as the evaluation of intensity of IL-4 and IFN- $\gamma$ in immunized offspring (TCD4+ IL-4, $p=0.8064$ and IFN- $\gamma, p=0.5606$; CD19+ IL-4, $p=0.7640$ and IFN- $\gamma, p=0.1302$; Fig. 3E-F and 5E-F).

\section{Interleukin 10 production and $B / T$ cell cooperation are necessary for $\mathrm{Th} 2$ regulation induced by maternal immunization}

Based on our previous publication about B10 cell involvement [19], we sought to investigate the IL-10 involvement by subjecting IL-10 $10^{-/}$mice to a maternal/neonatal immunization protocol and evaluating the offspring with the same parameters as WT mice. The maternal immunization of IL-10 ${ }^{-/-}$mice induced an increase in the TCD4+IL-4+ $(p=0.0111)$ percentage in 3 d.o. offspring without influencing the production intensity ( $p=0.5783$; Fig. 6A). During the same period, no influences on offspring B cells were observed (IL-4+, $p=0.7491$; IL-4 MFI, $p=0.1436$; Fig. 6B).

After neonatal immunization, offspring from immunized mothers, compared with offspring from non-immunized mothers, showed a reduction in TCD4+IFN- $\gamma+$ cells $(p=0.0405)$ and a reduction in the IL-4 intensity of TCD4+ cells ( $p=0.0053$, Fig. 6C). Furthermore, maternal immunization augmented IFN- $\gamma+(p=0.0419)$ and reduced IL-4+ $(p=0.0155)$ B cells of immunized offspring (Fig. 6D). No differences were observed in the intensity of IL-4 and IFN- $\gamma$ production (IL-4, $p=0.3143$ and IFN- $\gamma$, $p=0.8273$; Fig. 6D).

$\mathrm{CD} 28^{-/-}$mice are unable to produce $\operatorname{IgE}$ in response to allergen sensitization, as shown by the Th1-driven immunity with the predominance of IFN- $\gamma$ production, and because its $\mathrm{B} / \mathrm{T}$ cell cooperation is compromised [20]. Thus, we evaluated the effects of maternal immunization in $\mathrm{CD} 28^{-1-}$ mice and observed that maternal immunization induced augmented IL-4 intensity in TCD4+ cells $(p=0.0276$, Fig. 7A). 

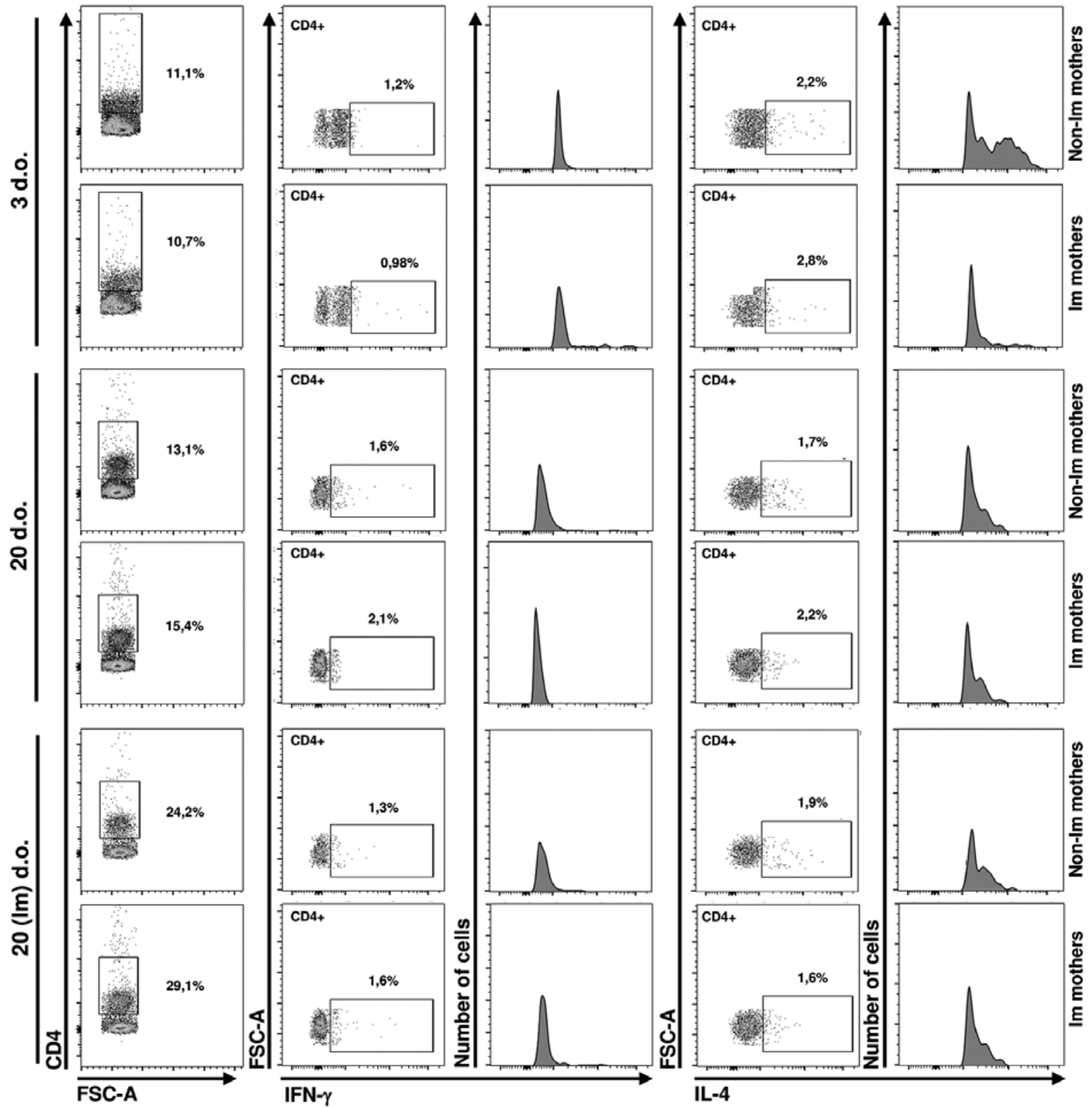

Fig. 2. Illustrative dot plots of the effect of maternal immunization on offspring cytokine production by TCD4+ cells. Each sample was acquired using the singlet cell gate (determined by FSC-A/FSC-H parameters) and then the lymphocyte gate (determined by their relative size/granularity); samples were then acquired gated as CD4+ cells (left panels) and isotype control based cytokine gating to evaluate percentage and intensity in each evaluation period and experimental group was performed accordingly, as indicated in the right panels

This effect was maintained after neonatal immunization ( $p=0.0370$, Fig. $7 \mathrm{C}$ ), and we observed a simultaneous increase in the IFN- $\gamma$ percentage $(p=0.0051)$ and intensity $(p=0.0005)$ in TCD4+ (Fig. 7C) and IFN- $\gamma$ intensity $(p=0.0055)$ in $\mathrm{B}$ cells of 3 d.o. offspring derived from immunized mothers (Fig. 7D).

\section{Discussion}

Our group has already reported that the maternal immunization protocol with OVA in WT mice could down-regulate the intracellular IL-4 production on offspring, suggesting a Th2 inhibition [14], and four years later, we also observed that maternal immunization with 

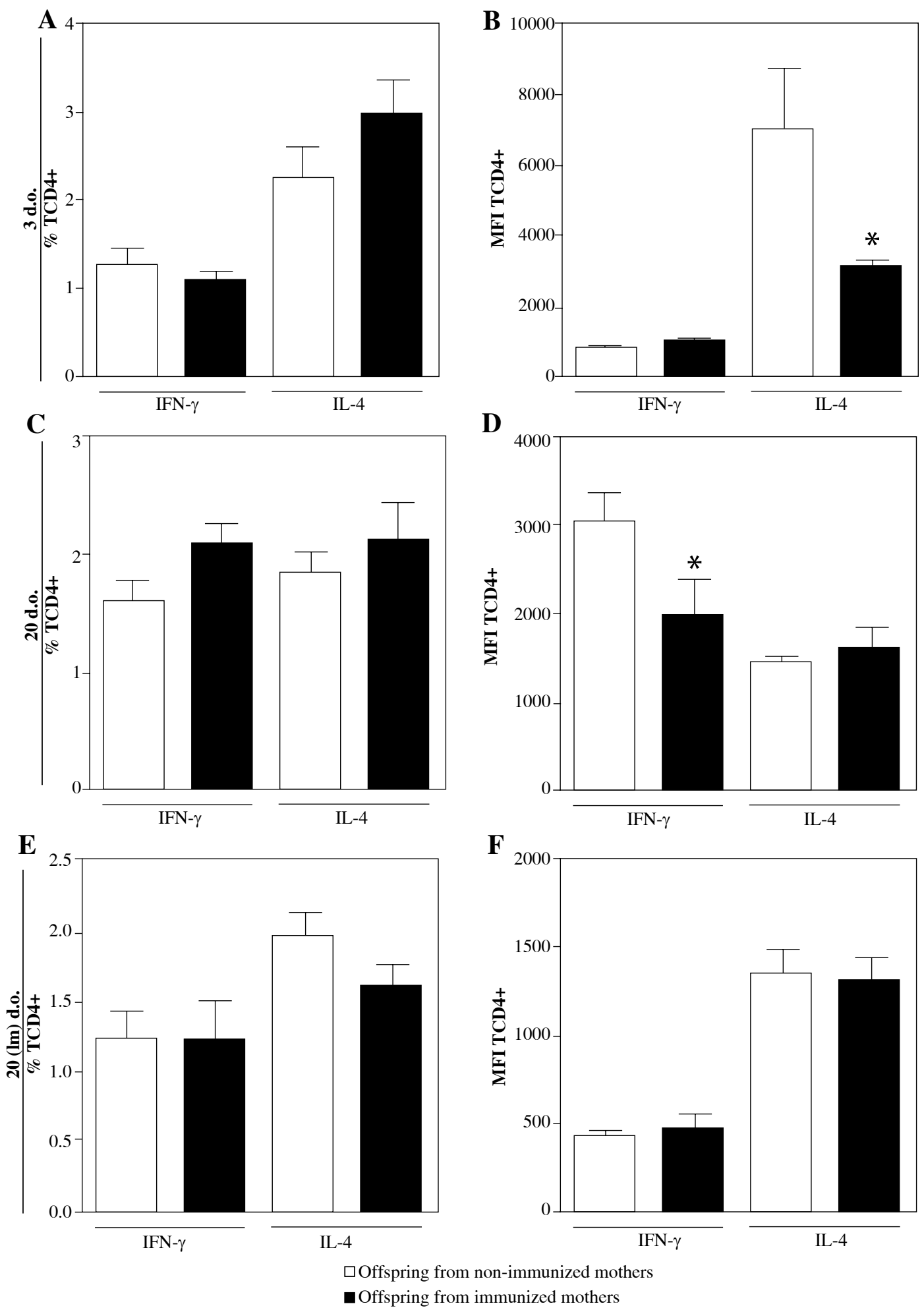

Fig. 3. Intracellular cytokine production in offspring T cells. WT female mice were immunized with OVA, boosted 10 and 20 days later and mated at day 21 with WT males. Offspring from immunized $(n=12)$ or non-immunized mothers $(n=12)$ were evaluated at 3 (3 d.o., $\mathbf{A}$ and $\mathbf{B})$ and 20 d.o. (20 d.o., $\mathbf{C}$ and $\mathbf{D})$ or immunized with OVA at 3 d.o., boosted at 13 d.o., and evaluated at 20 d.o. (20 [Im] d.o., E and F). Intracellular IFN- $\gamma$ and IL-4 percentage and intensity (MFI) were evaluated in offspring TCD4+ T cells. The data are presented as the means \pm SEM of each group. $* p<0.05$, compared with the control group 

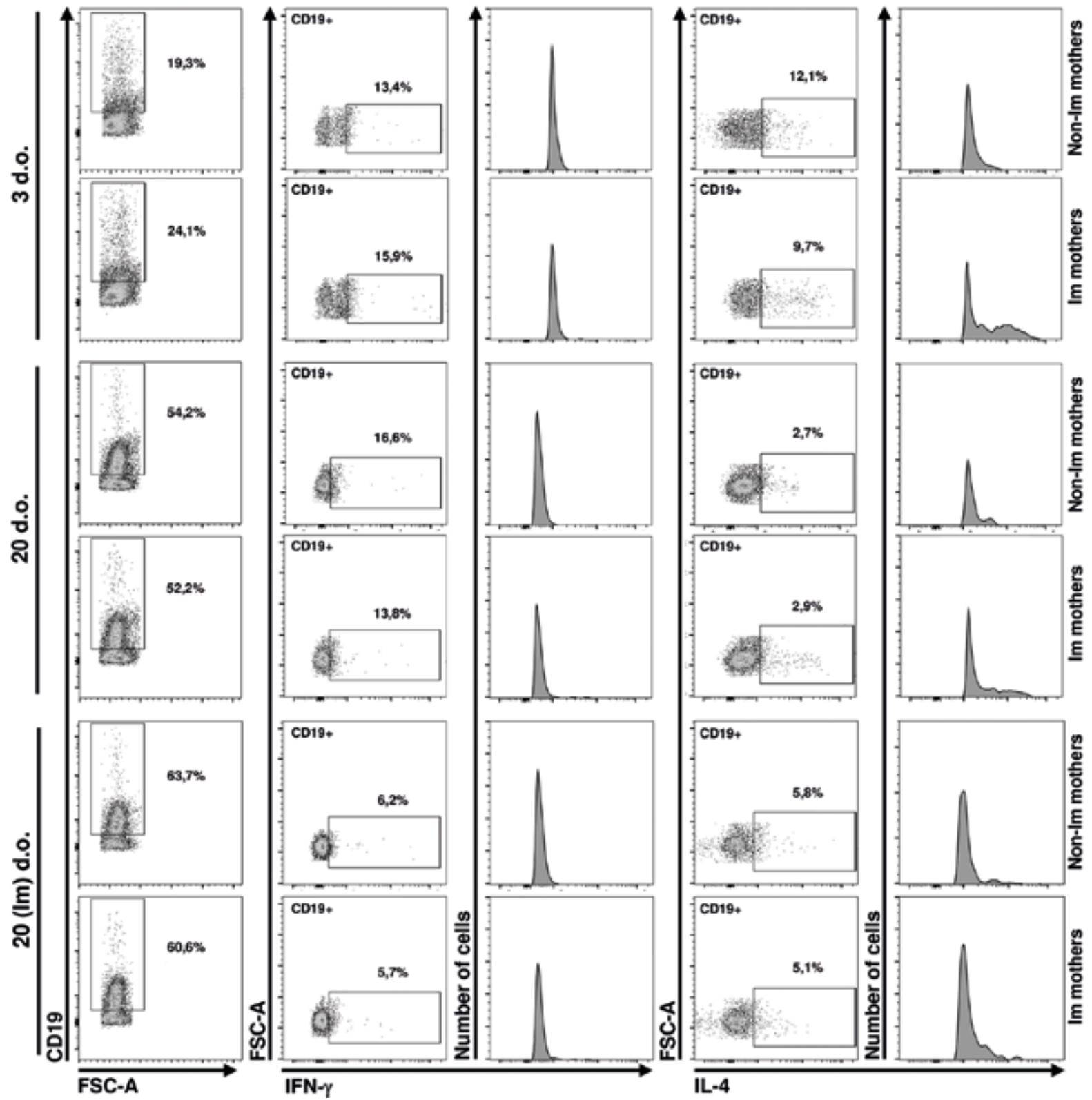

Fig. 4. Illustrative dot plots of the effect of maternal immunization on offspring cytokine production by B cells. Each sample was acquired using the singlet cell gate (determined by FSC-A/FSC-H parameters) and then the lymphocyte gate (determined by their relative size/granularity); samples were then acquired gated as CD19+ cells (left panels) and isotype control based cytokine gating to evaluate percentage and intensity in each evaluation period and experimental group was performed accordingly, as indicated in the right panels

a dust mite extract (Dermatophagoides pteronyssinus) could not induce the same cytokine and phenotype profile in offspring cells [7].

Recently, using the same experimental protocol adopted in the present work, we observed that WT mice derived from OVA-immunized mothers cannot develop pulmonary allergy inflammation in response to the same allergen [19]. This work reveals that maternal immunization can sup- press WT offspring IgE production and pulmonary inflammation, due to regulatory $\mathrm{B} 10$ cell induction in offspring.

Together, our previous observations raised some new questions, and it became necessary to evaluate the in vivo contribution of IL-10 and B/T cell collaboration to offspring allergy inhibition.

To assess the in vivo role of IL-10 in this mechanism, IL- $10^{-/-}$mice were subjected to the same maternal and neo- 

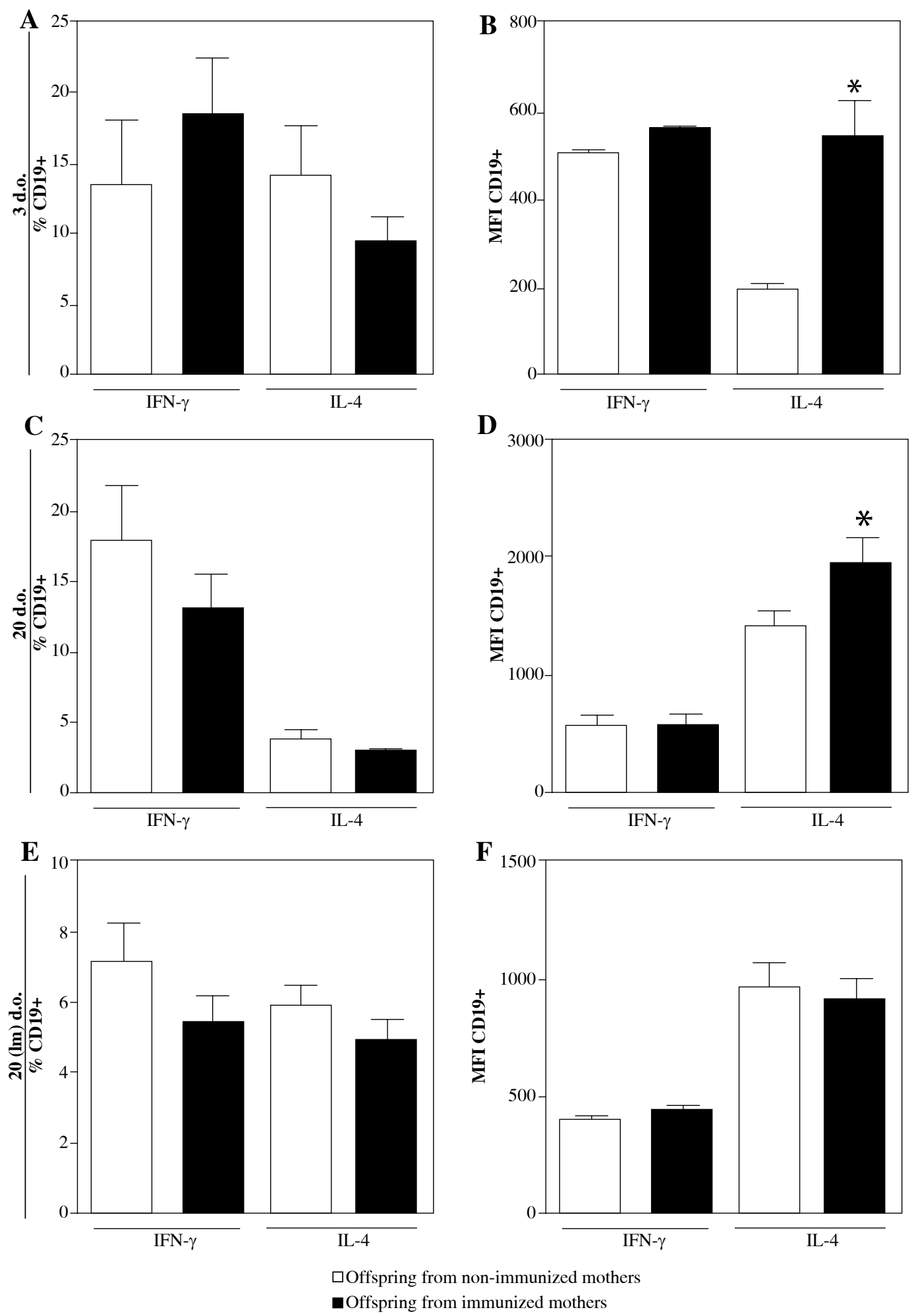

Fig. 5. Intracellular cytokine production in offspring B cells. WT female mice were immunized with OVA, boosted 10 and 20 days later and mated at day 21 with WT males. Offspring from immunized $(n=11)$ or non-immunized mothers $(n=11)$ were evaluated at 3 (3 d.o., A-B) and 20 d.o. (20 d.o., C-D) or immunized with OVA at 3 d.o., boosted at 13 d.o., and evaluated at 20 d.o. [20 (Im) d.o., E-F]. Intracellular IFN- $\gamma$ and IL-4 percentage and intensity (MFI) were evaluated in offspring CD19+ B cells. The data are presented as the means \pm SEM of each group. $* p<0.05$, compared with the control group 

in vivo influence of interleukin 10 and B/T cell collaboration
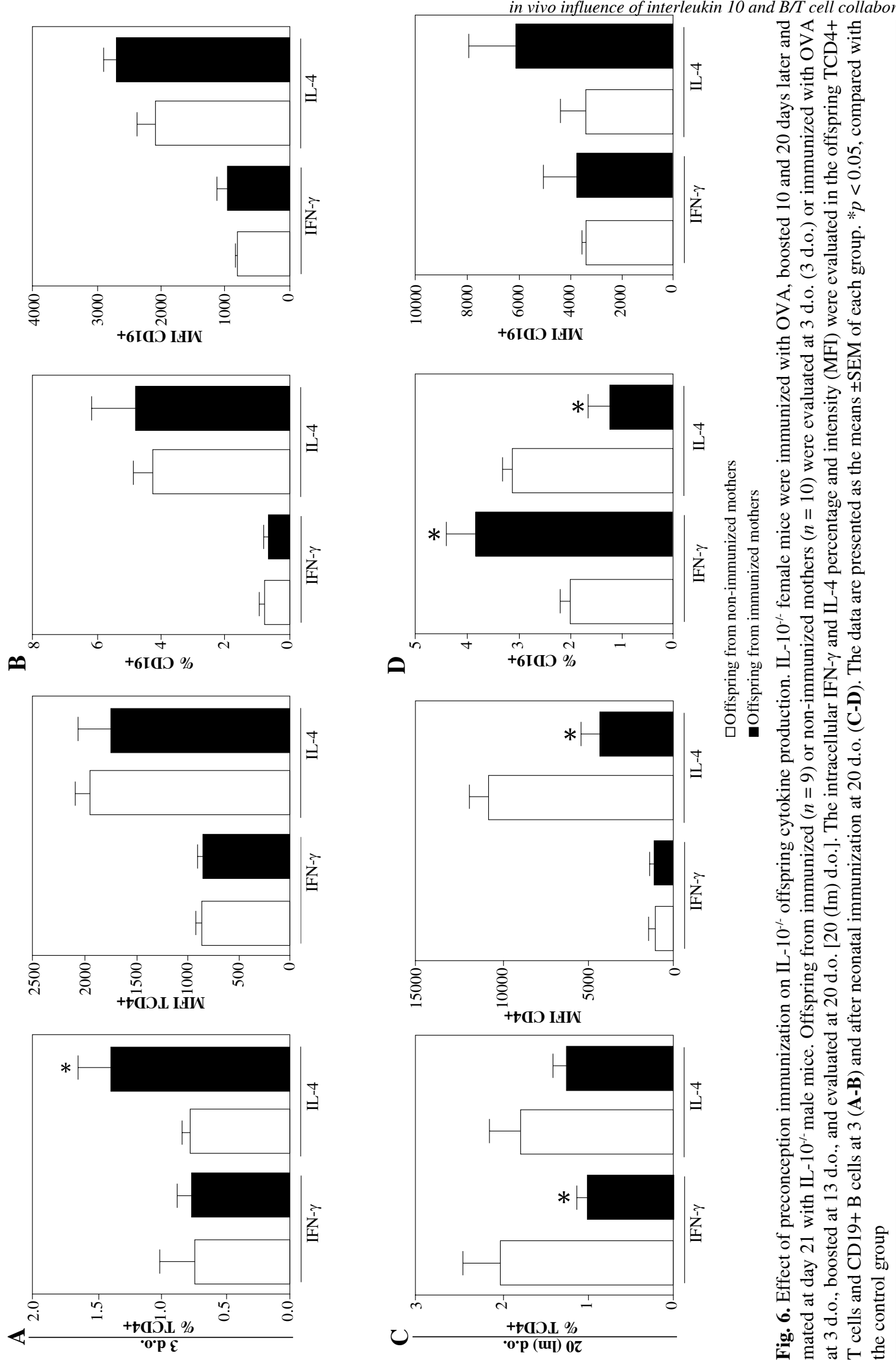

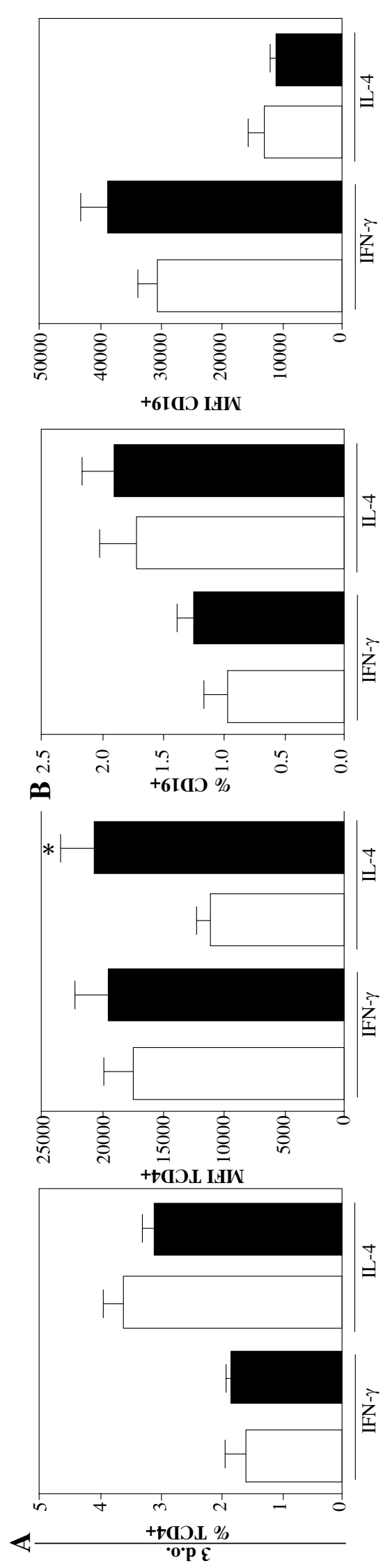
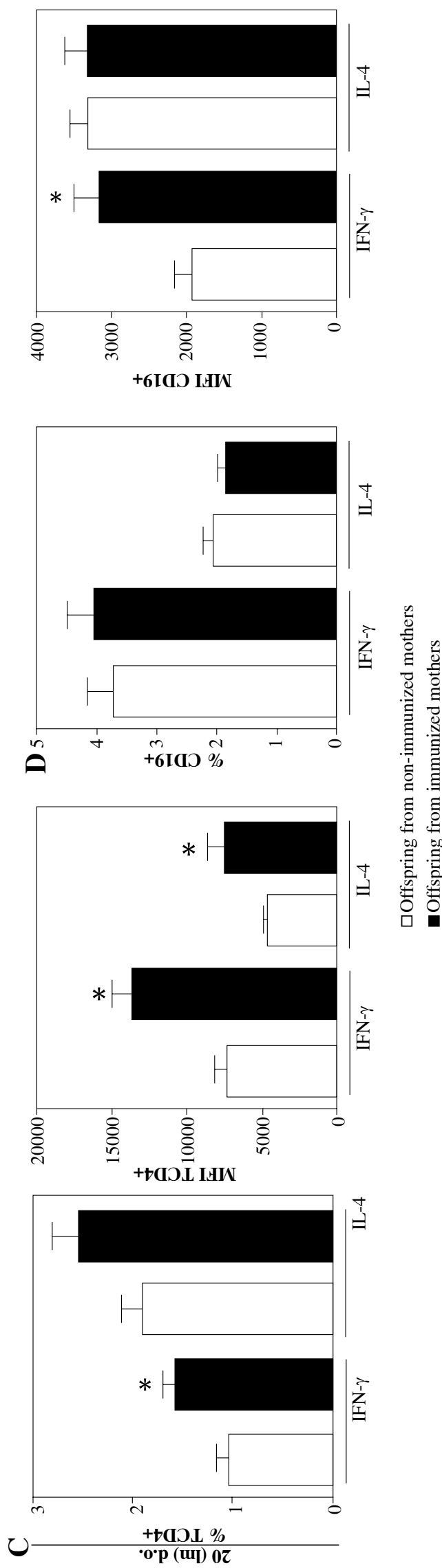

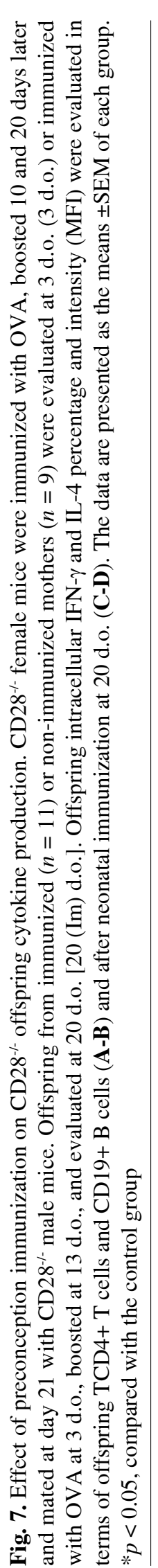


natal immunization protocol with OVA, and the absence of IL-10 resulted in an increased percentage of TCD4+ cells that produced IL-4 in offspring from immunized mothers. Since this increase was not observed in WT mice, it suggests a loss of regulation following Th2 responses in offspring. After neonatal immunization, IL-10 ${ }^{-/}$offspring from immunized mothers showed downregulation upon Th1/Th2 cytokine production, in contrast to WT mice.

These observations suggest that IL-10 is necessary to induce the regulatory effect of maternal immunization on offspring cells as well as to maintain the Th1/Th2 balance in immunized offspring. This data are in accordance with our recent evidence that maternal immunization exerts its inhibitory effect on offspring mainly by B10 cell induction [19].

Yoshizaki and collaborators used an autoimmunity model to show that $\mathrm{B} 10$ cell induction and regulatory functions depend on cognate B/T cell interactions [22]. Moreover, our model uses an allergen to induce protection, which cannot ensure that an $\mathrm{IgE}$ response has been induced in mothers. Based on that, we decided to subject CD28mice to a maternal/neonatal immunization protocol.

The CD $28^{-/-}$mice showed impaired T-cell responses and a reduced capacity to generate germinal centers $[23$, 24]. For B cells, CD28 deficiency compromised antibody class-switching, producing low-to-undetectable IgE levels and high levels of $\operatorname{IgM}$ and $\operatorname{IgG}$ antibodies in response to immunization [25].

We observed that the maternal immunization of CD28mice, similar to IL- $10^{-/-}$mice, could not down-regulate the Th2 profile in offspring as observed in WT mice. It is interesting to note that, in both CD28 $8^{-/-}$and $\mathrm{IL}-10^{-/-}$mice, the augmented IL-4 production in B cells observed in WT offspring from immunized mothers was lost.

The IL-4 production augments in B cells could be related to B10 cell induction since this population is characterized by autologous IL-4 production to exert its functional activity [15], thus suggesting that B10 cell induction is possible in both IL-10 and CD28 knockout mice.

These results reinforce the role of B10 cell induction as a mechanism for the regulation of allergy in offspring. Considering that in this same experimental model it has been described that the induction of B10 cells can be mediated by maternal IgG and that this seems to occur also in human B cells [19], the data generated in this work can support the future development of regulatory therapies for the development of allergies that are mediated by passive antibody transfer as recently proposed by the "MatIgG primary modulation theory" hypothesis [26].

In conclusion, our results reveal, complementarily to previous observations of our group, that offspring allergy and Th 2 cytokine profile inhibition mediated by maternal immunization with OVA seems to be dependent on IL-10 and efficient $\mathrm{B} / \mathrm{T}$ cell collaboration in vivo.

\section{Acknowledgments}

This study was funded through a grant from the Laboratory of Medical Investigation-56, Medical School, University of Sao Paulo, Sao Paulo, Brazil (LIM-56 HCFMUSP), grants \#2015/17256-3 and \#2018/05181-7 from the Săo Paulo Research Foundation (FAPESP), and grant \#115603/2015-8 from The National Council for Scientific and Technological Development (CNPq).

\section{The authors declare no conflict of interest.}

\section{References}

1. Dang VD, Hilgenberg E, Ries S, et al. (2014): From the regulatory functions of $\mathrm{B}$ cells to the identification of cytokine-producing plasma cell subsets. Curr Opin Immunol 28: 77-83.

2. Warner JA, Jones CA, Jones AC, et al. (2000): Prenatal origins of allergic disease. J Allergy Clin Immunol 105: S493498.

3. Fusaro A, Maciel M, Victor J, et al. (2002): Influence of maternal murine immunization with Dermatophagoides pteronyssinus extract on the type I hypersensitivity response in offspring. Int Arch Allergy Immunol 127: 208-216.

4. Fusaro AE, Brito CA, Victor JR, et al. (2007): Maternal-fetal interaction: preconception immunization in mice prevents neonatal sensitization induced by allergen exposure during pregnancy and breastfeeding. Immunology 122: 107-115.

5. Fusaro AE, de Brito CA, Taniguchi EF, et al. (2009): Balance between early life tolerance and sensitization in allergy: dependence on the timing and intensity of prenatal and postnatal allergen exposure of the mother. Immunology 128: e541-e550.

6. Rigato PO, Fusaro AE, Victor JR, et al. (2009): Maternal immunization to modulate the development of allergic response and pathogen infections. Immunotherapy 1: 141-156.

7. Lira AA, de Oliveira MG, de Oliveira LM, et al. (2014): Maternal immunization with ovalbumin or Dermatophagoides pteronyssinus has opposing effects on Fc gamma RIIb expression on offspring B cells. Allergy Asthma Clin Immunol 10: 47.

8. Muniz BP, Victor JR, Oliveira LdM, et al. (2014): Tolerogenic microenvironment in neonatal period induced by maternal immunization with ovalbumin. Immunobiology 219: 377-384.

9. Sgnotto FDR, Oliveira MG, Lira AAL, et al. (2017): Low doses of $\mathrm{IgG}$ from atopic individuals can modulate in vitro IFN- $\gamma$ production by human intra-thymic TCD4 and TCD 8 cells: an IVIg comparative approach. Hum Vaccin Immunother 13: $1563-1572$.

10. Victor JR (2014): Influence of maternal immunization with allergens on the thymic maturation of lymphocytes with regulatory potential in children: a broad field for further exploration. J Immunol Res 2014: 780386.

11. Futata E, de Brito C, Victor J, et al. (2006): Long-term anergy in orally tolerized mice is linked to decreased B7.2 expression on B cells. Immunobiology 211: 157-166.

12. Bento-de-Souza L, Victor JR, Bento-de-Souza LC, et al. (2016): Constitutive expression of genes encoding notch receptors and ligands in developing lymphocytes, nTreg cells 
and dendritic cells in the human thymus. Results Immunol 6: $15-20$.

13. Victor J, Fusaro A, Duarte A, et al. (2003): Preconception maternal immunization to dust mite inhibits the type I hypersensitivity response of offspring. J Allergy Clin Immunol 111: 269-277.

14. Victor JR, Muniz BP, Fusaro AE, et al. (2010): Maternal immunization with ovalbumin prevents neonatal allergy development and up-regulates inhibitory receptor Fc gamma RIIB expression on B cells. BMC Immunol 11: 11.

15. Ding Q, Yeung M, Camirand G, et al. (2011): Regulatory B cells are identified by expression of TIM- 1 and can be induced through TIM-1 ligation to promote tolerance in mice. J Clin Invest 121: 3645-3656.

16. Amu S, Saunders SP, Kronenberg M, et al. (2010): Regulatory B cells prevent and reverse allergic airway inflammation via FoxP3-positive T regulatory cells in a murine model. J Allergy Clin Immunol 125: 1114-1124.

17. Li J, Shen C, Liu Y, et al. (2015): Impaired function of CD5+CD19+CD1dhi B10 cells on IgE secretion in an atopic dermatitis-like mouse model. PLoS One 10: e0132173.

18. Mauri C, Bosma A (2012): Immune regulatory function of B cells. Annu Rev Immunol 30: 221-241.

19. de Oliveira MG, Oliveira LM, Lira AAL, et al. (2017): Preconception allergen sensitization can induce B10 cells in offspring: a potential main role for maternal IgG. Allergy Asthma Clin Immunol 13: 22.

20. Mathur M, Herrmann K, Qin Y, et al. (1999): CD28 interactions with either CD80 or CD86 are sufficient to induce allergic airway inflammation in mice. Am J Respir Cell Mol Biol 21: 498-509.

21. Freer G, Rindi L (2013): Intracellular cytokine detection by fluorescence-activated flow cytometry: basic principles and recent advances. Methods 61: 30-38.

22. Yoshizaki A, Miyagaki T, DiLillo DJ, et al. (2012): Regulatory B cells control T-cell autoimmunity through IL-21-dependent cognate interactions. Nature 491: 264-268.

23. Ferguson SE, Han S, Kelsoe G, et al. (1996): CD28 is required for germinal center formation. J Immunol 156: 45764581.

24. Lenschow DJ, Herold KC, Rhee L, et al. (1996): CD28/B7 regulation of Th1 and Th2 subsets in the development of autoimmune diabetes. Immunity 5: 285-293.

25. Borriello F, Sethna MP, Boyd SD, et al. (1997): B7-1 and B7-2 have overlapping, critical roles in immunoglobulin class switching and germinal center formation. Immunity 6: 303313.

26. Victor JR (2017): Allergen-specific IgG as a mediator of allergy inhibition: Lessons from mother to child. Hum Vaccin Immunother 13: 507-513. 